\title{
Degradation of PVC insulated cable by Small Punch test considering molecular weight change
}

\author{
Koga Yasutomo $^{1}$, Arao $_{\text {Yoshihiko }}{ }^{1}$, and Kubouchi Masatoshi ${ }^{1}$
}

${ }^{1}$ School of Chemical Science and Engineering, Tokyo Institute of Technology, Meguro-ku, Tokyo 152-8550, Japan

\begin{abstract}
PVC used as insulation material for industrial power cable was tried to evaluate degradation by Small Punch test (SP test) as a simple and easy assessment method. It was suggested that the SP test can analyze chemical changes from the correlation with IR. Qualitative assessment shows agreement between SP test and tensile test, and it is possible to apply to evaluation of strength at long aging times. However, the failure load in the SP test samples is complex and scattered, so that detailed study on SP test is required. Thus, we focused chemical reaction mechanisms and tried to clarify their influence on SP behavior. Molecular weight change was measured for each aging time by SEC and discussed how polymer chains are cut based on IR result. We discuss the relationship between molecular weight and the mechanical properties of PVC by SP test. The effectiveness of SP test as the evaluation method for residual life prediction will be addressed.
\end{abstract}

\section{Introduction}

PVC is widely used for insulation material of electric cable because of low cost, flexibility and stability. Lifetime of such insulation material for electric cables largely depends on condition of surrounding environment they located in $\left[\begin{array}{ll}1,2 & 2\end{array}\right.$. Although lifetime prediction of electric cables especially used in infrastructure is important, it may still be difficult. So we need lifetime estimation based on the easy and simple assessment method. Now, in this research, the Small Punch test (SP test) was tried to be introduced as a simple assessment method to evaluate the durability of PVC for electric cables. SP test is generally used in an evaluation of toughness of metal [3-5]. Load at the break-point by means of a small-ball pressing against a sample (Fig. 1) is measured. SP test is expected to be able to assess durability by minimum destructive test for infrastructure facility, because SP test can evaluate by very small disk shaped samples. If SP test is able to assess mechanical property of PVC resin effectively, lifetime prediction based on a minimum destructive test is possible.

In this study, sample as model material of "jacket" used in PVC cables is made and their change of mechanical property due to thermal degradation in ambient condition at different temperature is evaluated by tensile mode. The effectiveness of SP test is conducted by comparing tensile test with dumb-bell type specimen.

Chemical reaction as thermal degradation was also studied. Degradation behavior is analyzed by IR and SEC, and mechanism is discussed based on migration of plasticizers, dehydrochlorination and oxidation point view. The influence of chemical reaction on SP behavior is studied.

\section{Materials and methods}

\subsection{Tensile test and SP test}

Formulation of the model is shown in Table 1. Tensile test (dumbbell shape) and SP test (disk type speciment with diameter $6 \mathrm{~mm}$, thickness $1 \mathrm{~mm}$ ) was conducted by thermal degraded samples. The SP testing equipment is shown in Fig. 1 where the upper hole size is $3 \mathrm{~mm}$ and the ball diameter is $2 \mathrm{~mm}$. Testing speeds in Tensile and $\mathrm{SP}$ test are $50 \mathrm{~mm} / \mathrm{min}$ and $1 \mathrm{~mm} / \mathrm{min}$ respectively.

\subsection{Chemical reaction analysis}

Molecular weight change was analyzed by Size Exclusion Chromatography (SEC). THF was used as SEC eluent (sample 5mg / THF 25mL). The chemical structure change was analyzed by FT-IR.
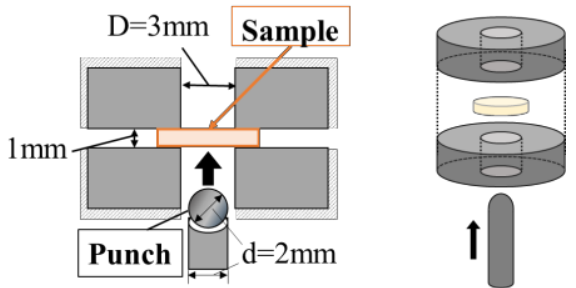

Figure 1. Schematic view of the SP test. 
Table 1. Formulation of the PVC cable jacket sample

\begin{tabular}{cc}
\hline Formulation & Jacket Mass [\%] \\
\hline PVC & 51.0 \\
\hline $\begin{array}{c}\text { Plasticizer } \\
\text { (DINP) }\end{array}$ & 26.5 \\
\hline $\begin{array}{c}\text { Stabilizer } \\
\text { (Ca-Zn based and Non lead) }\end{array}$ & 2.0 \\
\hline $\begin{array}{c}\text { Filler } \\
\text { (Heavy calcium carbonate) }\end{array}$ & 20.4 \\
\hline
\end{tabular}

\section{Results and discussion}

\subsection{Tensile test and SP test}

Fig. 2 is the load-displacement curves of SP test at $120{ }^{\circ} \mathrm{C}$. These curves bend near $2.3 \mathrm{~mm}$ regardless of exposure time. The SP testing ball diameter is $2 \mathrm{~mm}$, so this stroke position suggests that the deformation mode may change from the part of globe shape to the cylindrical shape as pulling-up to the cylinder axis direction. Comparing these four curves, as aging time is longer, the initial slope is larger and the length of the break is shorter. This shows that the sample is brittle.

The changes of elastic modulus after thermal aging by tensile test and SP test are shown in Figs. 3 and 4 . We define Elastic modulus in SP test as the slope of first period divided by sample thickness. In both case, Elastic modulus is increased exponentially. Increasing elastic modulus means the sample loses flexibility and becomes brittle. This result is consistent with that shown in Fig. 2. So we understand that dehydrochlorination and migration of plasticizer occurs. It is possible to assess qualitatively mechanical properties between tensile and SP test. But quantitatively Tensile test is larger than SP test. Because Tensile test is uniaxial tension, while SP test is triaxial tension when their testing is conducted. If we can construct damage modeling in Small Punch testing, we can predict life expectancy by using Arrhenius method.

\subsection{Chemical reaction analysis}

SEC can give the number-average molecular weight $(\mathrm{Mn})$ and weight-average molecular weight (Mw). The changes of them after degradation at 120 degree Celsius are shown in Fig. 5. The molecular weight change (Mw) becomes larger to about $500 \mathrm{~h}$, before Mw decreases. This suggests that at first reaction of polymer chain crosslinking is dominant and after about 400 hours, cut of chains is the main mechanism. Furthermore this time corresponds to the point of large change of elastic modulus in Figs. 3 and 4. So it is implied that molecular weight change of PVC resin is correlated with the mechanical properties of PVC.

The effectiveness of SP test as the residual life prediction method will be discussed on our presentation.

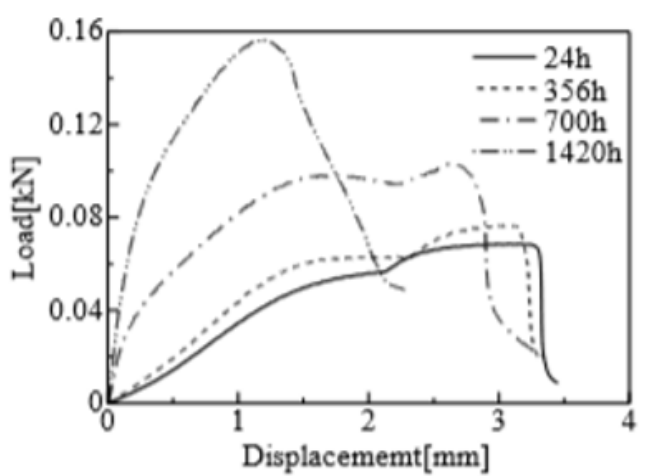

Figure 2. The load-displacement curve of the SP test at $120^{\circ} \mathrm{C}$.

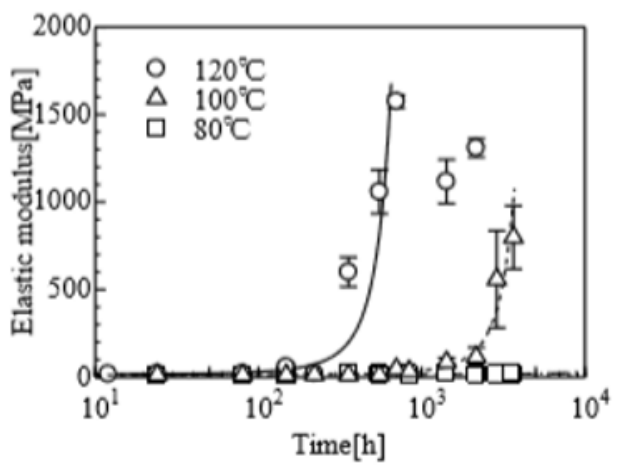

Figure 3. The change of Elastic modulus after thermal aging by tensile test.

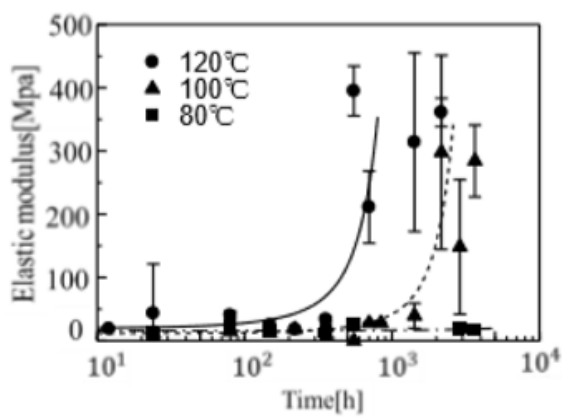

Figure 4. The change of Elastic modulus after thermal aging by SP test.

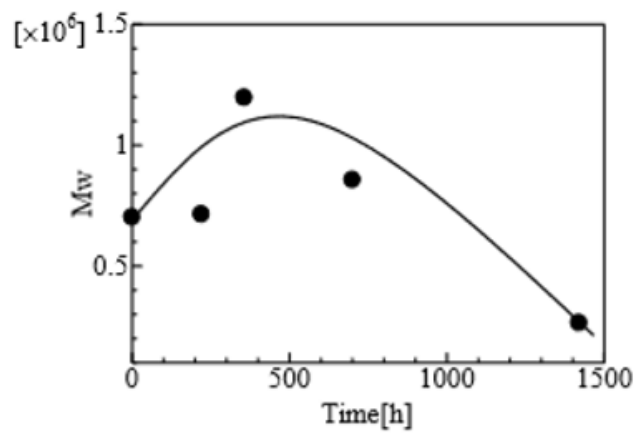

Figure 5. Effect of aging on $\mathrm{Mw}$ of the sample.

\section{Conclusion}

Qualitative assessment is agreed between SP test and tensile test, and the molecular weight change affect the mechanical properties of PVC by SP test. SP test is 
possible to apply to evaluation of strength in any aging time and predict the residual life. However, the load in the SP test sample is complex and scattered [6], so that detailed study on SP are required not only in stress distribution but also chemical reaction mechanism furthermore.

\section{References}

[1] P. Quennehen, I. Royaud, et al., Determination of the aging mechanism of single core cables with PVC insulation, Polymer degradation and stability, 119, 2015, 96-104.

[2] M. Ekelund, et al., Long-term performance of poly (vinyl chloride) cable, Part 2: Mechanical and electrical performances, Polymer degradation and stability, 92, 2007, 617-629.

[3] C. Rodriguez, et al., Application of the miniature small punch test for the mechanical characterization of polymer materials, Theoretical and Applied Fracture Mechanics, 86, 2016, 78-83.

[4] E. Martinez-Paneda, et al., Damage modeling in Small Punch Test specimens, Theoretical and Applied Fracture Mechanics, 86, 2018, 51-60.

[5] T. E. Garcia, et al., Estimation of the mechanical properties of metallic materials by means of the small punch test, Journal of Alloys and Compounds, 582, 2014, 708-717.

[6] R. J. Lancaster, et al., Modelling the small punch tensile behavior of an aerospace alloy, J. Materi als Science and Technology, 33, 2017, 1065-107 3. 